\title{
COHERENCE ENHANCEMENT DIFFUSION USING ROBUST ORIENTATION ESTIMATION
}

\author{
Mohammad A. U. Khan ${ }^{1}$, Tariq M. Khan ${ }^{2}$, Wadee Al-Halabi ${ }^{3}$, Hiba

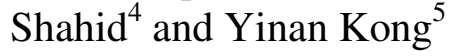 \\ ${ }^{1,4}$ Department of Electrical and Computer Engineering, \\ Effat University, Jeddah, Saudi Arabia. \\ mohammad_a_khan@yahoo.com \\ ${ }^{2,5}$ Department of Engineering, Macquarie University, Sydney, Australia \\ tariq045@gmail.com \\ ${ }^{3}$ Department of Computer Science, King Abdulaziz University, Saudi Arabia \\ wsalhalabi@kau.edu.sa
}

\begin{abstract}
In this paper, a new robust orientation estimation for Coherence Enhancement Diffusion (CED) is proposed. In CED, proper scale selection is very important as the gradient vector at that scale reflects the orientation of local ridge. For this purpose, a new scheme is proposed in which pre calculated orientation, by using orientation diffusion, is used to find the correct true local scale. From the experiments it is found that the proposed scheme is working much better in noisy environment as compared to the traditional Coherence Enhancement Diffusion.
\end{abstract}

\begin{abstract}
KEYWORDS
Fingerprint Image Enhancement, Coherence Enhancement Diffusion Directional Filter Bank (DFB), Decimation free Directional Filter Bank (DDFB).
\end{abstract}

\section{INTRODUCTION}

Biometric technology deals with identifying humans with their traits or characteristics. It deals with measurable albeit distinctive characteristics which can be used to label and to a large extent describe individuals [1]. Examples include, face recognition, DNA, palm print, hand geometry, retina, iris recognition and fingerprint. Out of these many biometric techniques, fingerprint identification is the most prevailing one which has been successfully used in numerical applications in the past [2]. It has been observed that every human being has a unique and immutable fingerprint [3]. Individually is hidden in the pattern of ridges and valleys on the fingerprint surface. Ridges are found to terminate themselves at some locations and split at others. These are referred to as ridge ending and bifurcation respectively. The locations corresponding to these ridge endings and bifurcations are known as minutiae points. Identifying human fingerprint means locating correctly these minutia points.

With the advancement in computer technology, need to automatic fingerprint identification of fingerprint scans arises. Reliable extraction of minutiae is one of the critical step for an automatic fingerprint identification system (AFIS). We can forsee the importance of the quality of input David C. Wyld et al. (Eds) : CCSIT, SIPP, AISC, PDCTA, NLP - 2014 
fingerprint scan in determining the reliability of the overall automatic fingerprint identification system (AFIS) [1]. The fingerprints are obtained with fingerprint scanners that may not provide a perfect scan in all circumstances. The presence of dirt or oil on the surface of the finger may result in blurred scan. Furthermore, due noise present in scanner electronics, the fingerprint scans may not be of clear quality. Mostly, the noise in fingerprint image manifest itself in terms of cuts or interpolated ridge lines. For automatic fingerprint identification system (AFIS) to work reliability, these broken ridge lines need to be restored vis some enhancement process [4].

A fingerprint enhancement process generally consists of estimating local image parameters, which in turn are used to tune a contextual filter that moves from one location to another on the surface of fingerprint, smoothing noise on the way. Contextual filters can be conveniently defined in the framework of partial differential equations [5]. A major development in PDE based image diffusion was introduced earlier by Perona and Malik [6]. On the basis of their study, number of diffusion filters have been proposed [7, 8, 9, 10, and 11]. Most of these techniques are based on a scalar diffusivity parameter which guides the diffusion flux moving along the orthogonal to the gradient direction. These techniques are linear and isotropic in nature, and have same common disadvantages, like edge blurring. To improve the performance of these isotropic diffusion Joachim Weickert [12] proposed a non-linear isotropic diffusion, which replaces scalar diffusivity parameter with diffusion matrix to provide greater flexibility in dealing with oriented structure on point to point basis. This technique is well known as Coherence Enhanced Diffusion (CED). The analysis of the CED technique can be carried out by an eigen decomposition of the diffusion matrix, which results in eigenvalues and eigenvectors. The eigenvalues dictates the amount of smoothing, while the eigenvectors are responsible for steering these smoothing in proposer directions. Though eigenvalues are essential part of coherence enhanced diffusion, a clean computation of eigenvectors without noise may be ultimately responsible for evolving the whole diffusion process. We believe that a CED in its current status lacks this clean steering process.

The impact of steering diffusion in wrong directions may distort some genuine minutia points while creating fake ones as the same time. We propose to relieve the stress in providing clean eigenvectors in the form of embedding an orientation diffusion block in the CED process. Thus letting CED to worry only about proper diffusion speeds at each point. Thus CED will provide the diffusion of noisy fingerprints with diffusivity parameters that align themselves with clean directions at each diffusion step.

The research presented in this paper is concerned with finding reliable orientations and then finding a way to embed them in the conventional CED algorithm. Orientation diffusions [13] has been there for a while, that provides a framework for diffusing directions alone with noise reduction at each step. We adapted the method to be used with the conventional CED scheme to boost its performance in noisy images. The rest of the paper is organized as follow. Section II details the Coherence Enhanced Diffusion. In section III a summary of orientation diffusion method is discussed. Section IV describes how the pre-computed orientation is used in Coherence Enhancement Diffusion. In Section V testing and validation is presented and finally the paper is concluded in section VI.

\section{COHERENCE ENHANCED DIFFUSION}

Weickert [14] considered a general class of anisotropic diffusion process. The process utilizes a diffusion matrix depending on the local image structure via the so-called second-moment matrix (or structure tensor). The second-moment matrix is a well-established tool for texture analysis $[15,15,17$, and 18]. In [14], Weickert investigated the use of second-moment matrix information to design anisotropic diffusion scale-space, which enhances the coherence of the ridge-like 
texture in fingerprint. The method is generally referred to as Coherence Enhanced Diffusion (CED), and is summarized in the following sections.

Consider an input fingerprint image $L(x, y)$. The anisotropic scale-space for the image $L(x, y)$ can be found by implementing the heat equation, given by:

$\partial_{t} L=\operatorname{div}(D \nabla L)$

where $D$ is the $2 \times 2$ diffusion matrix, adopted to the local image structure, via a structural descriptor, called the second-moment matrix $\mu$, defined as:

$\mu=\left(\begin{array}{ll}\mu_{11} & \mu_{12} \\ \mu_{12} & \mu_{22}\end{array}\right)=\mu=\left(\begin{array}{cc}L_{x}^{2} & L_{x} L_{y} \\ L_{x} L_{y} & L_{y}^{2}\end{array}\right)$

where $L_{x}{ }^{2}, L_{x} L_{y}$ and $L_{y}{ }^{2}$ represents the Gaussian derivative filters, in x-and-y directions. The matrix is a symmetric $2 \times 2$ matrix, with two dominant eigen values $\lambda_{\min }$ and $\lambda_{\max }$, given by:

$\lambda_{\text {min }}=1 / 2\left(s_{11}+s_{12}+\alpha\right)$
$\lambda_{\text {max }}=1 / 2\left(s_{11}+s_{12}-\alpha\right)$

Where

$\alpha=\sqrt{\left(s_{11}-s_{22}\right)^{2}+4 s_{12}^{2}}$

Let us now define the diffusion speeds $c_{u}$ and $c_{v}$ as

$$
\begin{aligned}
& c_{u}=\max \left(0.01,1-e^{-\left(\lambda_{\min }-\lambda_{\max }\right)^{2}} / k^{2}\right) \\
& c_{v}=0.01
\end{aligned}
$$

The diffusion matrix $D$ with component is given by:

$$
\begin{aligned}
& d_{11}=1 / 2\left(\left(c_{u}-c_{v}\right)+\frac{\left(c_{u}-c_{v}\right)-\left(s_{11}-s_{22}\right)}{\alpha}\right) \\
& d_{12}=\frac{\left(c_{u}-c_{v}\right) s_{12}}{\alpha} \\
& d_{22}=d_{11}=1 / 2\left(\left(c_{u}-c_{v}\right)-\frac{\left(c_{u}-c_{v}\right)-\left(s_{11}-s_{22}\right)}{\alpha}\right)
\end{aligned}
$$


Once the diffusion matrix is constructed, we are set to start the evaluation process using Eq. 1 with input image $L(x, y)$ as the starting point. In the process of evaluation, the image got enhanced ridge pattern, as shown in Fig. 1

The diffusion matric $\left(\begin{array}{ll}d_{11} & d_{12} \\ d_{12} & d_{22}\end{array}\right)$ can be decomposed using eigen decomposition, into eigenvalues $c_{u}$ and $c_{v}$ along with eigen directions. By plotting these eigen directions on top of the enhanced image it can be observed that these directions need to be corrected for better evolution. However, their cleaning process for eigen directions need to be embedded in the CED process such that each diffusion step is proceeded in the clean direction. For cleaning directions at each step, we take the help of orientation diffusion process as suggested in [19].

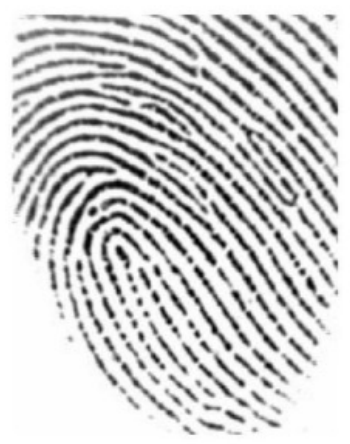

(a)

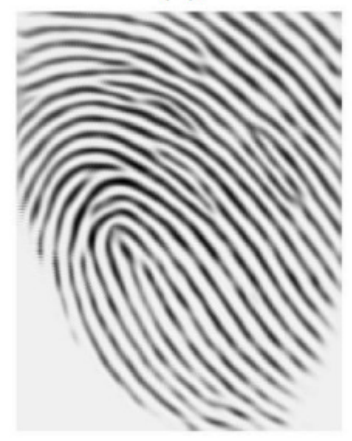

(c)

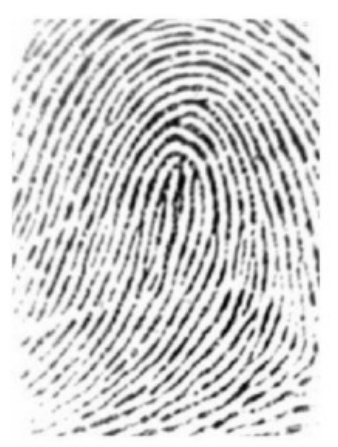

(b)

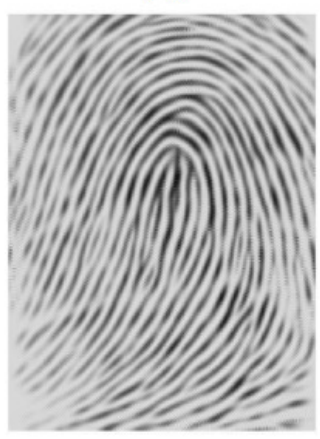

(d)

Fig. 1. a) and b) are two noisy images from FVC database. c) and d) shows the results of CED enhancement of a) and b) respectively.

\section{ORIENTATION DIFFUSION}

Orientation filed estimation is considered as first step moving towards enhancement process as suggested in [1]. But in noisy environment, this provide a challenge. In research literature, different techniques have been in use for orientation estimation in noise-degraded images. The notable examples can be cited in [20], where a filter bank based approach is used, and [21], where waveform projection, and [22] with spectral estimation. To increase the degree of accuracy for the initial coarse estimation, mathematical modelling using Bayesain network [23] and complex polynomial can be proposed. Later, [24] introduces an enhanced gradient-based approach for reliable orientation estimation. In this scheme, dominant orientation of a base block from its four overlapping neighbourhoods is estimated and finally a best estimate is selected from least noise- 
affected neighbourhood. However, gradients are unl-reliable and error-prone on fingerprint images affected by noise like scares, smudges, dryness or wetness of finger [25]. Recently, DFB has [26] also been used to provide reliable orientation. However, we feel that orientation diffusion provide a natural coupling for our purpose. The reason for this natural coupling stems from the fact that orientation diffusion provides a gradually decrease in noise levels present in orientations as opposed to a step process adopted by DFB based orientations.

According to [19], the correct way of diffusing orientation is to adopt a non-linear diffusion function in the form as given below:

$\theta(x, t+1)=\theta(x, t)+\lambda \sum_{n \in N(x)} \sin (\theta(n, t)-\theta(x, t))$

where $N(x)$ is the set of all 8 neighbours around $\mathrm{x}$ location. Also $\theta$ has to be in the range of $[-\pi, \pi]$.

The discrete implementation of orientation diffusion, as described by Eq. 7 on a cropped noisy pattern of the fingerprint image shown in Fig. 4. The initial condition of diffusion process was calculated from the gradient of the image intensities, i.e, $\theta_{\text {initial }}=\tan ^{-1}\left(\frac{g_{y}}{g_{x}}\right)+\frac{\pi}{2}$. The boundary condition are set to zero gradients.

The results of running the diffusion may be seen in Fig. 2. We notice the desirable effect of smoothing orientations on the way. The two singularities of orientations that ate present in the fingerprint would be difficult to detect in the original image using a local gradient operator. We found that this orientation diffusion inspired by simple physical systems, can de-noise an orientations data effectively, by eating out already existing print-singularities with new generation of singularities, Thus, it does support the casuality property of diffusion.

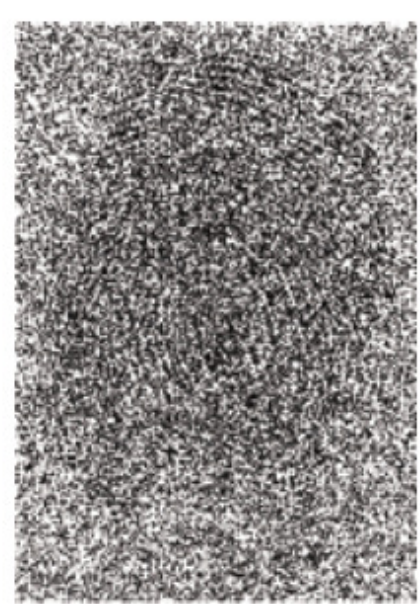

a

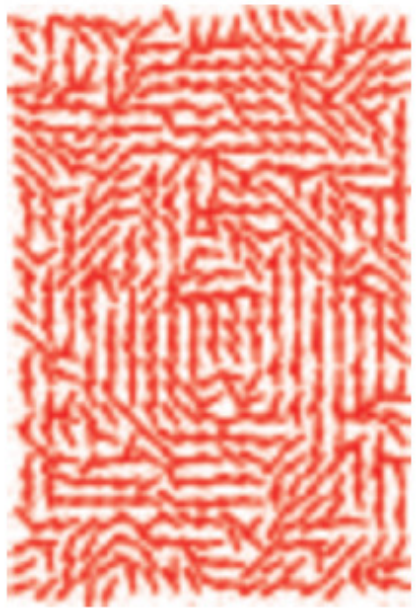

b

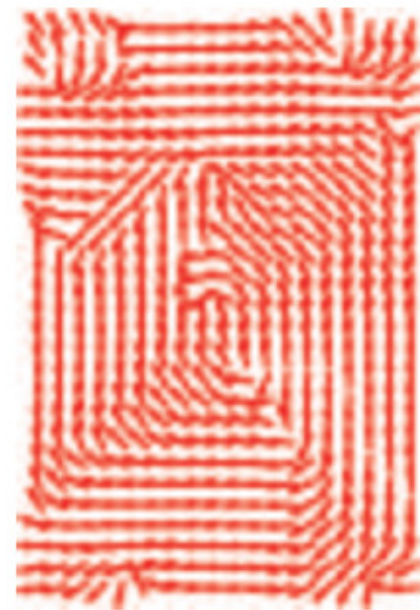

C

Fig. 2. a) sample noisy image, b) CED Orientation, and c) Orientation enhanced by orientation diffusion 


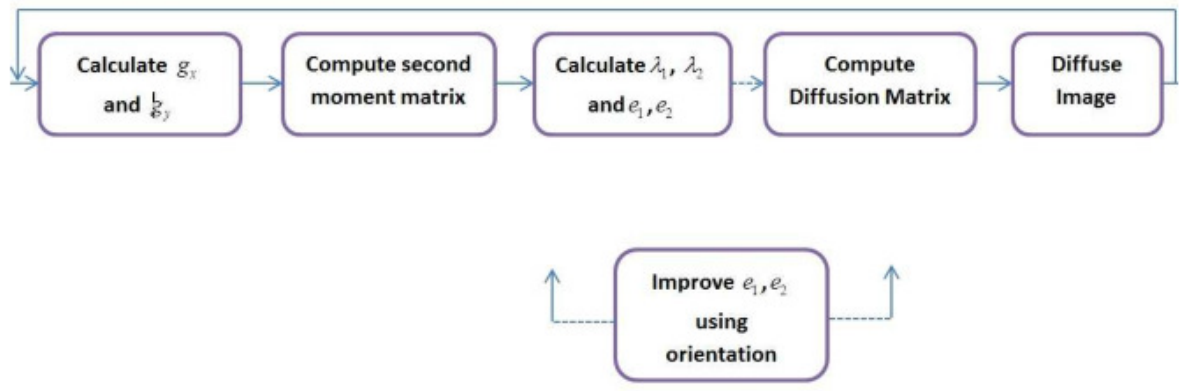

Fig. 3. Orientation Fed CED block diagram

\section{ORIENTATION FED COHERENCE ENHANCED DIFFUSION}

As we discussed in section II, the CED process starts by taking an input image, then calculate its gradients $g_{x}$ and $g_{y}$ respectively. From $g_{x}$ and $g_{y}$, it computes the second moments matrix $\left(\begin{array}{ll}s_{11} & s_{12} \\ s_{12} & s_{22}\end{array}\right)$ to depict the rotation in local structure. The second moment matrix can be decomposed to provide us with eigen values $\lambda_{1} \& \lambda_{2}$ and also the eigen directions $e_{1} \& e_{2}$. In the conventional design, these lambdas and eigenvectors can be used in the construction of diffusion matrix used in diffusion process.

However, in our proposed scheme, we keep intact the lambdas, but take $e_{1} \& e_{2}$ direction for further cleaning. For this purpose, we feed $e_{1} \& e_{2}$ into our orientation diffusion module for further de-noising. Once cleaned, we feed this cleaned $e_{1} \& e_{2}$ back to our module of computing diffusion matrix. And thus the process continues.

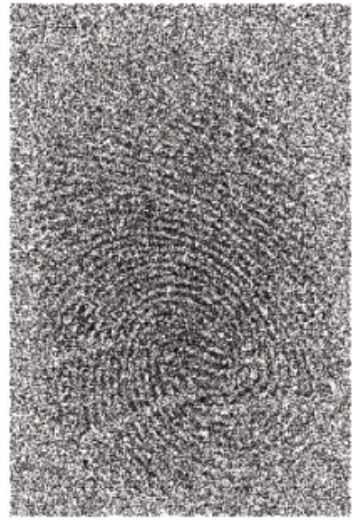

a

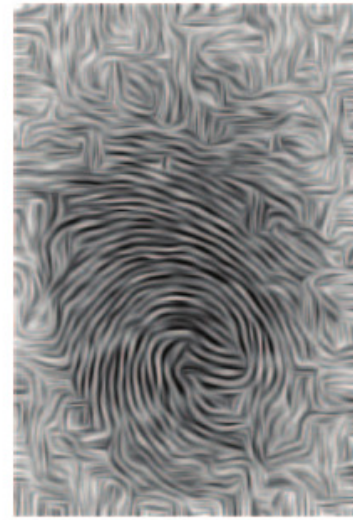

b

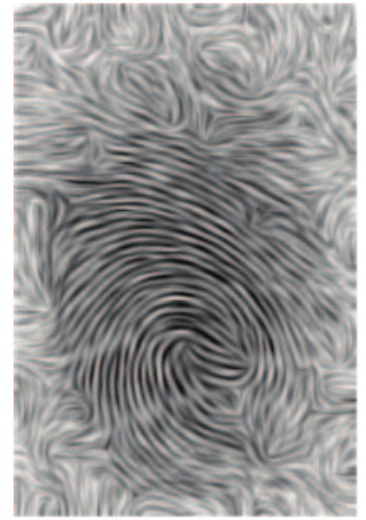

C

Fig. 4. a) sample noisy image, b) CED enhanced image, and c) Orientation Fed CED 


\section{TESTING AND VALIDATION}

Statistically, the set of minutiae denoted by symbol $M$ were algorithmically obtained and evaluated against the set of minutiae marked by an expert denoted by symbol $F$ and the minutiae are classified into two types: termination and bifurcation. Each minutiae point $m$ compartmentalized into one of the following classes:

- Correct: The correlation between minutiae points generated through the algorithm and those obtained from the expert is perfect position, i.e $\left(m \in F \cap M\right.$ and type $_{F}(m)=$ type $\left._{M}(m)\right)$

- False: The expert did not mark the location corresponding to the one obtained through the algorithm, i.e $(m \in M, m \notin F)$

- Missing: The minutiae location has been marked by the expert but couldn't be seen within the algorithmically generated image, i.e $(m \notin M, m \in F)$

- Misclassified: The minutiae location marked by the expert could be is also the one found by the method with the exception that one has been classified as bifurcation and other has been classified as termination, i.e $\left(m \in F \cap M\right.$ and type $_{F}(m) \neq$ type $\left._{M}(m)\right)$

Compartmentalization of all the minutiae set is followed by the performance measurement which is computed statistically. This is done on minutiae obtained from the algorithm as well as minutiae marked by the experts which makes the performance measurement relative to either total number of minutiae found algorithmically or the total number of minutiae found by the expert markings. The measures relative to minutiae marked by the expert which detects the aptitude of the technique to detect correct minutiae are as follows:

- Missing minutiae is defined as $\frac{M i s \sin g}{|F|}$

- False minutiae is defined as $\frac{\text { False }}{|F|}$

- Misclassified minutiae is defined as $\frac{\text { Missclassified }}{|F|}$

- Correct minutiae is defined as $\frac{\text { Correct }}{|F|}$

- Total error which is the summation of false positive, false negative and misclassified errors is defined as $\frac{\text { Mis } \sin g \cup \text { False } \cup \text { Missclassified }}{|F|}$

- Classification rate for the purpose of enhancing the graphical visualization is defined as Correct

Missclassified 
Large number of missing minutiae could be tolerated to certain extent but the large number of false minutiae would lead to larger noise rate within the mapping stage. On the other hand, prominence of classification error is based on the manner in which the mapping algorithm deals with the types of minutiae. Using the techniques described above, the database of true minutiae was generated, by using the sample images given in Fig. 6 which is used to evaluate the orientation and scale-matched filters to produce improved input image along with the techniques described above.

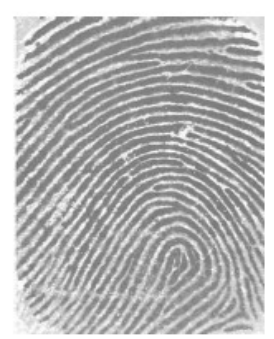

(Image 1)

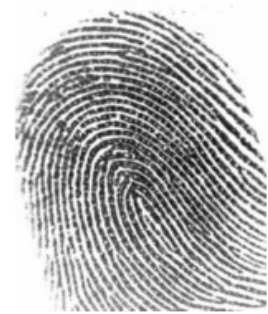

(Image5)

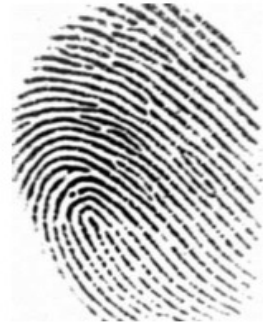

(Image2)

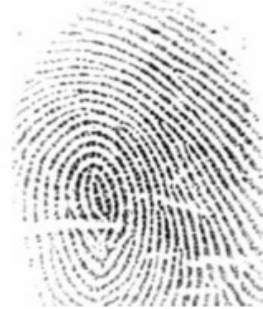

(Image6)

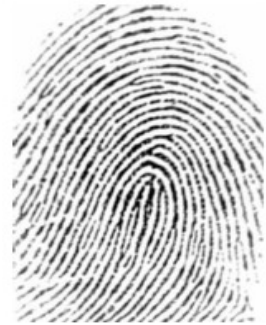

(Image 3)

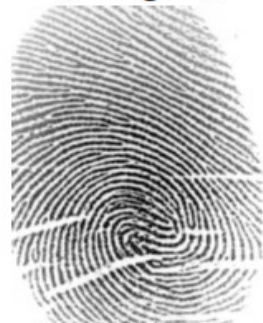

(Image7)

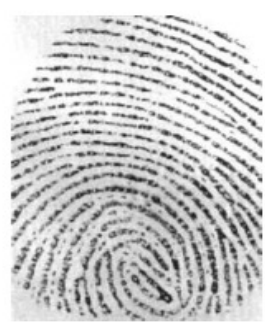

(Image4)

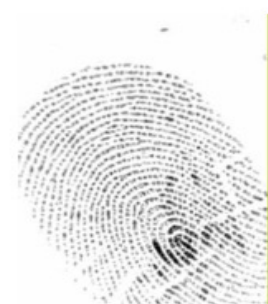

(Image8)

Fig. 5. Sample images used for evaluation measure

The table compares the result obtained using the above method.

Table1. This table shows the evaluation measure comparing minutiae of proposed algorithm

\begin{tabular}{|c|c|c|c|c|}
\hline Proposed & Correct & False & Missing & Misclassified \\
\hline Image1 & 21 & 4 & 6 & 4 \\
\hline Image2 & 18 & 8 & 8 & 5 \\
\hline Image3 & 21 & 19 & 9 & 1 \\
\hline Image4 & 31 & 5 & 5 & 1 \\
\hline Image5 & 20 & 12 & 20 & 3 \\
\hline Image6 & 10 & 15 & 20 & 4 \\
\hline Image7 & 16 & 11 & 27 & 1 \\
\hline Image8 & 6 & 2 & 30 & 2.5 \\
\hline Mean & 17.875 & 9.5 & 15.625 & \\
\hline
\end{tabular}


Table2. This table shows the evaluation measure comparing minutiae of [12] CED Enhancement algorithm

\begin{tabular}{|c|c|c|c|c|}
\hline [12]CED & Correct & False & Missing & Misclassified \\
\hline Image1 & 19 & 8 & 6 & 6 \\
\hline Image 2 & 17 & 9 & 7 & 5 \\
\hline Image3 & 20 & 21 & 6 & 3 \\
\hline Image4 & 24 & 5 & 10 & 4 \\
\hline Image5 & 21 & 17 & 19 & 5 \\
\hline Image6 & 8 & 16 & 22 & 2 \\
\hline Image7 & 15 & 10 & 26 & 3 \\
\hline Image8 & 5 & 3 & 30 & 2 \\
\hline Mean & 16.125 & 13.25 & 15.75 & 3.75 \\
\hline
\end{tabular}

The average number of minutiae points present in the image marked by the expert and the ones marked by the algorithm for all the eight images was taken, which represented \$F\$. This was computed to be 15 using which some performance measures were computed. For the proposed method, classification error which is calculated as misclassified over correctly detected minutiae was found to be

$\frac{\text { Missclassified } / F \cap M}{|F|} * 100=0.89 \%$

For the CED method, classification error which is calculated as misclassified over correctly detected minutiae was found to be

$\frac{\text { Missclassified } / F \cap M}{|F|} * 100=1.45 \%$

In Fig7, the bar chart shows the performance evaluation measure of both schemes.

\section{CONCLUSIONS}

Coherence enhancement diffusion filters are the one which have control on the size/shape of the filter but, these use derivative for rotation matrix which is not considered as a reliable method. Therefore, in this work presents a new and a reliable method for orientation estimation is proposed to explicitly calculate the orientation field of a fingerprint image. By using this orientation new local scales for Diffusion matrix are adaptively calculated. The experiments conducted on the noisy images showed promising results of the proposed scheme. 


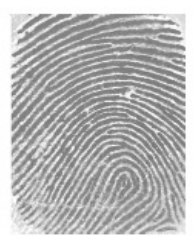

(a1)

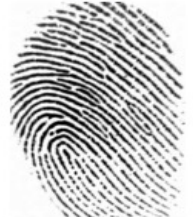

(a2)

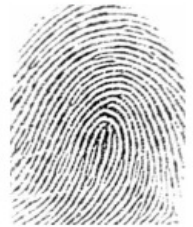

(a3)

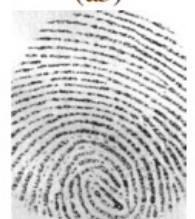

(a4)

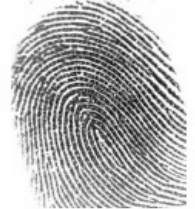

(a5)

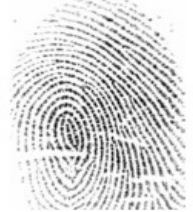

(a6)

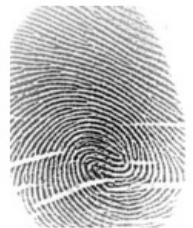

(a7)

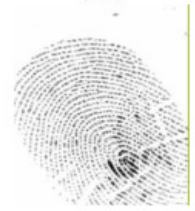

(a8)

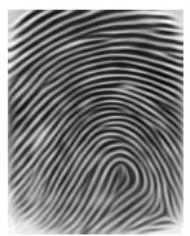

(b1)

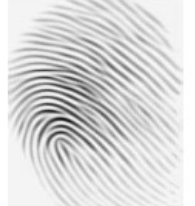

(b2)

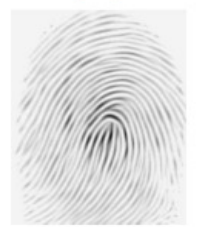

(b3)

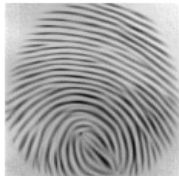

(b4)

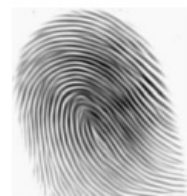

(b5)

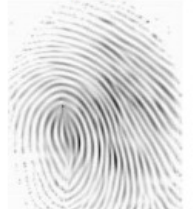

(b6)

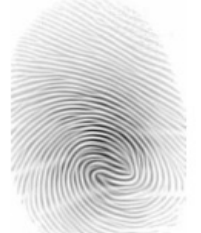

(b7)

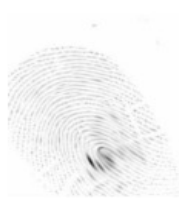

(b8)

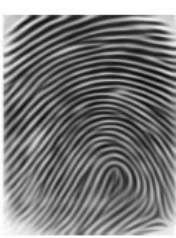

(c1)

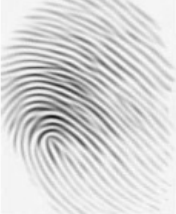

(c2)

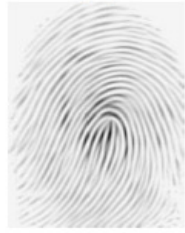

(c3)

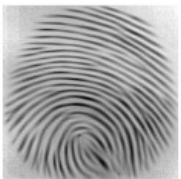

(c4)

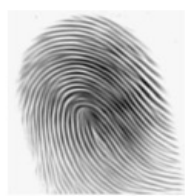

(c5)

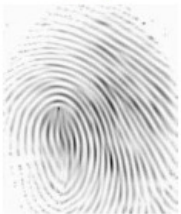

(c6)

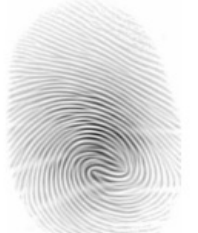

(c7)

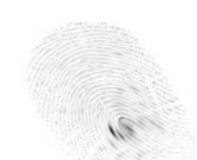

(c8)

Fig. 6. Sample images used for evaluation measure 


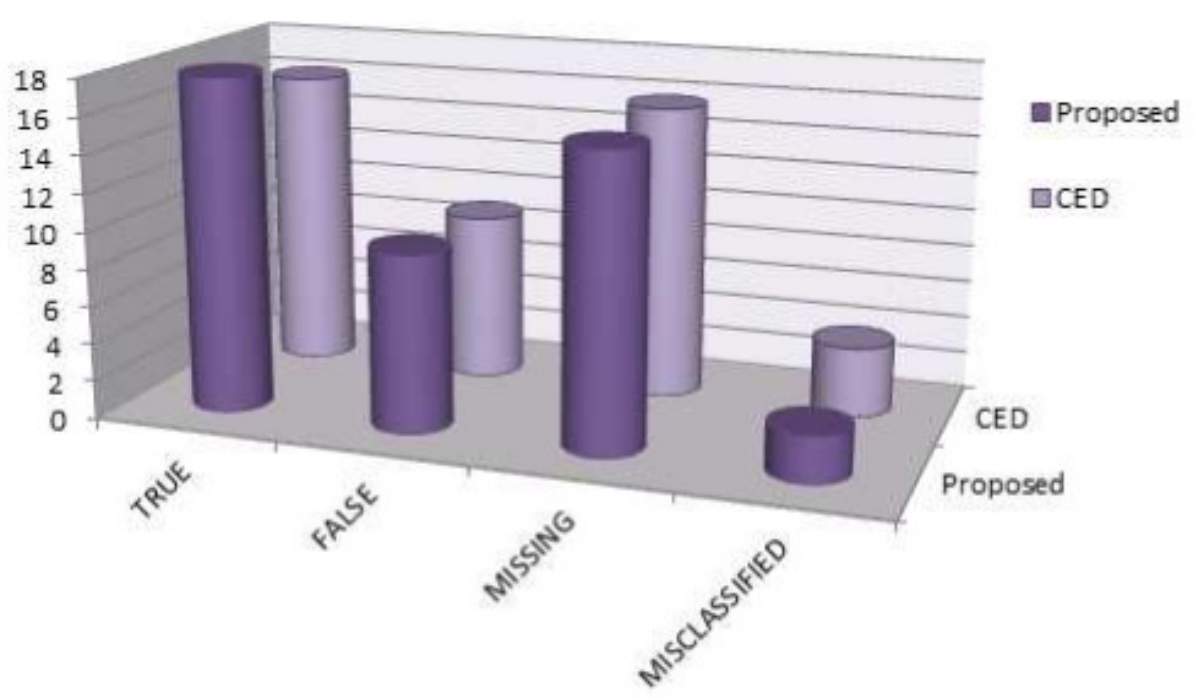

Fig. 7. Performance Evaluation Measure of both schemes

\section{REFERENCES}

[1] L. Hong, Y. Wan, and A. Jain, "Fingerprint image enhancement: Algorithm and performance evaluation," IEEE Transactions on Pattern Analysis and Machine Intelligence, vol. 20, pp. 777789, 1998.

[2] M. A. Khan amd A. Khan, T. Mahmmod, M. Abbas, and N. Muhammad, "Fingerprint image enhancement using principal component analysis (pca) filters," in International Conference on Information and Emerging Technologies (ICIET), 2010.

[3] Anil K. Jain, S. Prabhakar, and Sharath Pankanti., Can identical twins be discriminated based on fingerprints?, Michigan State University, De- partment of Computer Science, Michigan State University, East Lansing, Michigan, October 2000., technical report msu-cse-00-23, edition.

[4] M.A.U. Khan, T. M. Khan, and S. A. Khan, "Coherence enhancement diffusion using multi-scale dfb," in 7th International Conference on Emerging Technologies, 2011.

[5] J Weickert, "A review of nonlinear diffusion filtering," cale-Space Theory in Computer Vision, vol. 1252, pp. 3-28, 1997.

[6] P Perona and J Malik, "Scale-space and edge detection using anisotropic diffusion.," IEEE Trans Mach Intell., vol. 12(7), pp. 629639, 1990.

[7] L Alvarez, P-L Lions, and J-M Morel, "Image selective smoothing and edge detection by nonlinear diffusion,” II SIAM J Numer Anal, vol. 29, pp. 845866, 1992.

[8] G Sapiro, "Tannenbaum, affine invariant scale-space," International Journal of Computer Vision, vol. 11, pp. 25-44, 1993.

[9] L Rudin, S Osher, and E Fatemi, "Nonlinear total variation based noise removal algorithms," Modeliz Mat Traitement d Images, p. 149179, 1992.

[10] HM Salinas and D Cabrera Fernandez, "Comparison of pde-based nonlinear diffusion approaches for image enhancement and denoising in optical coherence tomography," IEEE Transactions on Medical Imaging, vol. 26, pp. 761-771, 2007.

[11] D Fang, Z Nanning, and X Jianru, "Image smoothing and sharpening based on nonlinear diffusion equation.," Signal Processsing, vol. 88(11), pp. 28502855, 2008.

[12] J Weickert, “Coherence-enhancing diffusion filtering," Int. J. Computer Vision,, vol. 31, pp. 111-127, 1999.

[13] Muhammad A. K. Khan and Wadee Alhalabi, "Robust multi-scale orientation estimation: Spatial domain vs fourier domain," in Inter- national Conference on Communications, Signal Processing, and their Applications, 2013. 
[14] Joachim Weickert, Anisotropic Diffusion in Image Processing, ECMI Series, Teubner-Verlag, Stuttgart, Germany, 1998.

[15] K. Ito and K. Kunisc, Augmented Lagrangian methods for nonsmooth, convex optimization in Hilbert spaces, Preprint No. 409/1994, Fachbere- ich Mathematik (3), Technische Universitat Berlin, Strae des 17. Juni 136, 10623 Berlin, Germany, 1994.

[16] R. Illner and J. Tie, "On directed diusion with measurable background," Math. Meth. Appl. Sci., p. 681690, 1993.

[17] O. Faugeras and R. Keriven, "On projective plane curve evolution," in ICAOS 96: Images, wavelets and PDEs, Lecture Notes in Control and Information Sciences, 1996, p. 6673.

[18] G.-H. Cottet and M. El Ayyadi, "Nonlinear pde operators with memory terms for image processing," in Proc. IEEE Int. Conf. Image Processing (ICIP96, Lausanne, Sept. 1619, 1996), 1996, p. 481483.

[19] P. Perona, “Orientation diffusions," IEEE Trans. on Image Process., vol. 7, pp. 457-467, 1998.

[20] A.K. Jain, S. Pankanti, and L. Hong, "A multichannel approach to fingerprint classification," IEEE Trans. Pattern Anal. Machine Intell, vol. 21, pp. 348359, 1999.

[21] B.G. Sherlock, D.M. Monro, and K. Millard, "Fingerprint enhancement by directional fourier filtering," IEE Proc. Vision, Image Signal Process., vol. 141, pp. 8794., 1994.

[22] C.L. Wilson, G.T. Candela, and C.I. Watson, "Neural network fingerprint classification," J. Artif. Neural Networks, vol. 1, pp. 203-228, 1994.

[23] S.C. Dass, "Markov random field models for directional field and singularity extraction in figerprint images," IEEE Trans. Image Process, vol. 13, pp. 13581367, 2004.

[24] Yi Wang, Jiankun Hu, and Fengling Han, "Enhanced gradient-based algorithm for the estimation of fingerprint orientation fields," Applied Mathematics and Computation, vol. 185, pp. 823833, 2007.

[25] Gottschlich C, P. Mihailescu, and A. Munk, "Robust orientation field estimation and extrapolation using semilocal line sensors,” IEEE Trans. Inf. Forensics Sec., vol. 4, pp. 802811, 2009.

[26] P. T. H. Truc, M. A. U. Khan, Y. K. Lee, and T. S. Kim, "Vessel enhancement filter using directional filter bank," Computer Vision and Image Understanding, vol. 113, pp. 101-112, 2009.

\section{AUTHORS}

\section{Dr Yinan Kong}

Dr Kong leads the VLSI Research Group at Macquarie. He is also currently the Director of Higher Degree Research, Department of Engineering, Macquarie University, Sydney, Australia. Dr Kong obtained his PhD from the University of Adelaide, Australia.

During his research at the VLSI Research Group at Macquarie University and Centre for High Performance Integrated Technologies and Systems (CHiPTec) at the University of Adelaide, Dr Kong focused on digital VLSI design at an arithmetic level for specific applications like cryptography and DSP. He achieved the hardware construction of a 1024-bit RSA cryptosystem at only $0.4 \mathrm{~ms}$ per decryption, the fastest ever FPGA implementation of RSA. In the meantime, he developed Bachelor Degree of Computer Engineering at Macquarie Engineering as well as postgraduate teaching in embedded computer system design and computer arithmetic with hardware focused.

\section{Tariq Mahmood Khan}

Tariq Mahmmod Khan is interested in both Digital Image Processing (with an emphasis on biometrics) and VLSI. He is currently involved in the hardware implementation of multi-modal biometrics project, led by Yinan Kong. The main goal of this project is to develop a fast processor for different design approaches. The aim is to test these approaches on a multi-modal biometric system consisting fingerprint, iris and face. Prior to starting his $\mathrm{PhD}$ in Engineering at Macquarie University, he received a degree in $\mathrm{BS}$ Computer Engineering from COMSATS Institute of Information \& Technology, Islamabad Pakistan and MSc in Computer Engineering from University of Engineering \& Technology, Taxila Pakistan.

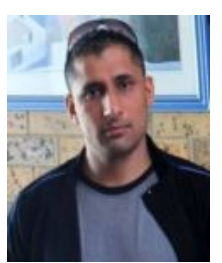

